\title{
Characterization of breast masses: a comparative study between automated breast ultrasound (ABUS) and digital breast tomosynthesis (DBT)
}

\author{
Lamiaa Mohamed Bassam Hashem', Reham Hussein Mohamed Ali², Maha Hussein Helal', \\ Emad ELdin E. L. Gemeae ${ }^{3}$ and Amr Farouk Ibrahim Moustafa ${ }^{2 *}$
}

\begin{abstract}
Background: Automated three-dimensional (3D) breast ultrasound (US) systems and breast tomosynthesis are promising breast imaging modalities. The study aims to compare the diagnostic indices of the 3D imaging techniques: digital breast tomosynthesis (DBT) and automated breast ultrasound (ABUS) in the characterization of breast masses. This prospective study included 32 women with breast masses either detected by means of clinical examination or with the mammographic exam. All of them have been subjected to tomosynthesis and automatic breast ultrasound examinations. The images from both modalities were then analyzed one at a time by two experienced representative radiologists in consensus. Results were compared to each other and to pathology and follow-up of typically benign findings

Results: The masses statistically evaluated in this study were 37 in number, among which 16 were benign and 21 were malignant. The sensitivity and specificity of tomosynthesis in the characterization of breast masses were 100\% and $81.25 \%$, respectively, while automated breast ultrasound were $100 \%$ and $75 \%$, respectively.

Conclusion: Tomosynthesis and automated breast ultrasound are two promising modalities in breast imaging. Their diagnostic indices in this study were very close to one another; therefore, they can be used as an adjunct modality to mammography for early diagnosis of breast cancer.
\end{abstract}

Keywords: Breast cancer, Automated breast 3D ultrasound, Breast tomosynthesis

\section{Background}

Breast cancer is the second leading cause of cancer demise in women [1]. Estimates indicate a nearly $40 \%$ breast cancer mortality reduction when screening women yearly beginning at age 40 [2]. Despite the fact that mammography is an outstanding screening modality in the recognition of early breast cancer, it is defective, especially for women with dense breasts. In women with dense breast tissue, the affectability of mammography is

\footnotetext{
* Correspondence: amrfaroukmoustafa@cu.edu.eg

${ }^{2}$ Department of Diagnostic and Interventional Radiology, National Cancer Institute, Cairo University, Cairo, Egypt

Full list of author information is available at the end of the article
}

decreased. Furthermore, women with dense breasts have an expanded danger of developing malignancy and cancers detected in them are larger and more frequently node-positive [3, 4].

Breast ultrasound, both handheld and automated, is powerful in distinguishing mammographically occult cancer in women with dense tissue. Ultrasound is essential for the characterization of breast masses. It is commonly employed as an adjunct to diagnostic clinical mammography and as an aid to screening mammography in dense breasts [5].

Automated three-dimensional (3D) breast ultrasound (US) systems and breast tomosynthesis are promising

\section{Springer Open}


modalities intended to defeat the inadequacies of handheld ultrasound (HHUS) being operator-dependent and take relatively a long time when compared to hand-held ultrasound, 3D-ABUS has been found to give comparable image quality and diagnostic information, BI-RADS ratings, and mass detection rates [6, 7].

Recent studies show that 3D breast tomosynthesis is a potential for increasing the sensitivity of breast cancer detection, especially for cancers manifesting as distortions or calcification [8].

The study aims to compare the diagnostic indices of the 3D imaging techniques: digital breast tomosynthesis (DBT) and automated breast ultrasound in the characterization of breast masses.

\section{Methods}

\section{Patients}

This prospective study was performed in the female imaging unit of specialized Cancer Institute and approved by its Research and Ethical committee; all the included cases gave informed consent.

The study was conducted on 32 patients presented to the clinic with either palpable breast mass or as a part of early screening between January 2017 and July 2017 . Their ages ranged from 25 to 74 years (mean age 52.97 $\pm 12.21 \mathrm{SD}$ years). Five patients had bilateral lesions and the total number of lesions was 37 lesions.

\section{Diagnostic tools}

All of the cases $(n=32)$ were subjected to both 3D tomosynthesis and Automated breast ultrasound.

\section{Tomosynthesis examination protocol design}

A craniocaudal (CC) and a mediolateral oblique (MLO) view DBT were obtained with the patient in a standing position using a DBT unit prototype. Breast compression was applied, similar to that of mammography. Images were acquired with a GE mammography system.

\section{Automated breast examination protocol design}

A layer of lotion is applied to the breast to ensure good contact between the 3D ABUS device and the skin. Then, a curved panel is placed over the breast and gently flattens the tissue against the body. Images were acquired using a GE Invenia ABUS system (GE Healthcare).

\section{Image analysis}

The tomosynthesis and automated ultrasound data were evaluated by two experienced radiologists in consensus; both observers were unaware of the pathological data of each patient. Each mass was evaluated regarding shape, margin, asymmetry, calcification, mass number, location, extension, skin thickening, and BIRADS classification were done.
Pathological results were used as the gold standard of reference for 32 out of the 37 masses, as for the remaining 5 masses, ultrasound proved these lesions to be simple cysts/fat-containing lesions (hamartoma).

\section{Statistical analysis}

Data were coded and entered using the statistical package SPSS (Statistical Package for the Social Sciences) version 24. Data were summarized using frequency (count) and relative frequency (percentage) for categorical data. Standard diagnostic indices including sensitivity, specificity, positive predictive value (PPV), and negative predictive value (NPV) were calculated. For comparing categorical data, chi-square $\left(\chi^{2}\right)$ test was performed. The exact test was used instead when the expected frequency is less than 5. A $p$ value of less than 0.05 was considered as statistically significant.

\section{Results}

This prospective study included a total of 32 patients presented to the NCI with breast masses (detected by clinical examination or by mammographic examination), their mean age was $52.97 \pm 12.21 \mathrm{SD}$.

Out of 32 patients, 37 masses were detected, 16 masses were benign while 21 masses were malignant. Five patients had bilateral lesions.

Pathological results were used as the gold standard of reference for 32 out of the 37 masses, as for the remaining 5 masses, ultrasound proved these lesions to be simple cysts $(n=3) /$ fat containing lesion (hamartoma) $(n=2)$.

The histological diagnosis in the patients included in this study is mentioned in Table 1 .

Each breast mass was evaluated by both tomosynthesis and automated ultrasound regarding the following criteria:

\section{The shape of the lesion}

Regarding the shape of the lesion, both modalities show agreement in 27 masses, 16 were irregular, 8 were oval, and 3 were rounded. On the other hand, 1 mass appear irregular in tomosynthesis but oval by automated ultrasound. No mass was detected by tomosynthesis in 1 case but was detected by automated images as irregular small masses (Fig. 1).

The relation between both modalities regarding the shape is shown in Table 2.

\section{Regarding the margin of the lesion}

Both modalities showed agreement in 11 cases with circumscribed masses and 13 cases with spiculated margin; on the other hand, significant mismatch appears in two cases in which appear as circumscribed by tomosynthesis while shown as indistinct/speculated by automated ultrasound (Table 3). 
Table 1 The histological diagnosis in the patients included in the study

\begin{tabular}{|c|c|c|c|}
\hline & & Count of lesions & $\%$ \\
\hline \multirow[t]{2}{*}{ Pathology } & Benign & 16 & 43.2 \\
\hline & Malignant & 21 & 56.8 \\
\hline \multirow[t]{10}{*}{ Pathology details } & Adenocarcinoma & 1 & 2.7 \\
\hline & Cyst & 4 & 10.8 \\
\hline & Duct carcinoma in situ & 1 & 2.7 \\
\hline & Fibroadenoma & 6 & 16.2 \\
\hline & Hamartoma & 2 & 5.4 \\
\hline & Invasive duct carcinoma & 14 & 37.8 \\
\hline & Invasive lobular carcinoma & 2 & 5.4 \\
\hline & Mixed invasive duct carcinoma and invasive lobular carcinoma & 3 & 8.1 \\
\hline & Mastitis & 2 & 5.4 \\
\hline & Metastases & 2 & 5.4 \\
\hline
\end{tabular}

\section{Mass number and location}

92.9\% of cases show agreement by both modalities in the presence of multiple masses,72.7\% agreement in the presence of single mass with statistically significant results $p$ value $<0.001$.
Regarding the location of the lesion, the largest number of masses was detected in the upper outer quadrant with $83 \%$ agreement by both modalities, 6 cases were detected retro areolar with $100 \%$ agreement (Table 4).
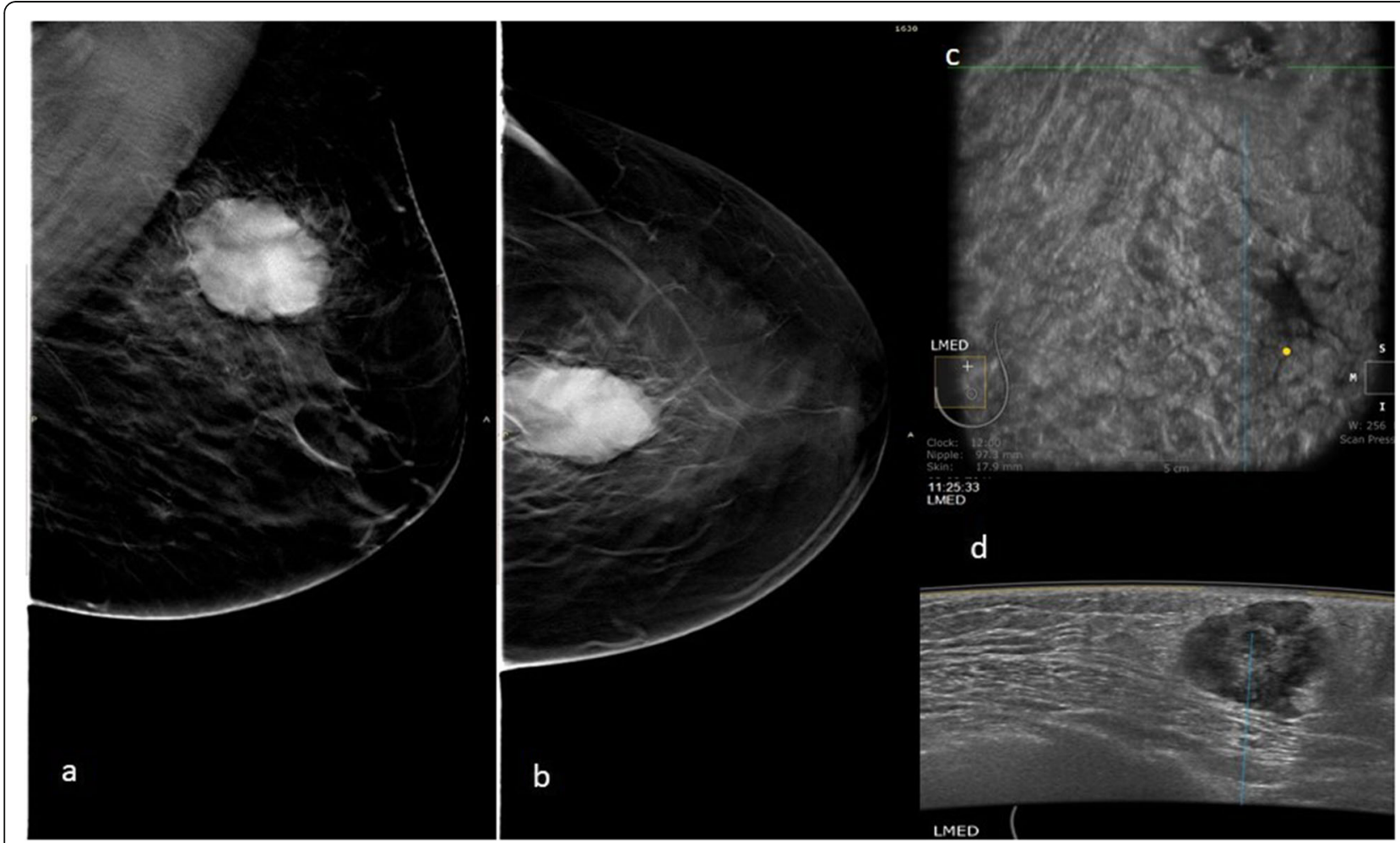

Fig. 1 A 51-year-old female patient was presented with a left UOQ breast lump. a, b Digital breast mammography tomosynthesis MLO and CC views of the left breast show an oval-shaped micro lobulated high-density mass at UOQ with no skin thickening (BIRADS IV). Automated ultrasound left medial coronal image (c) shows focal irregular defect with retraction phenomena at distance $97.3 \mathrm{~mm}$ from the nipple. Automated ultrasound left medial axial image (d) shows an irregular shaped mass with spiculated margin, non-parallel orientation, and heterogeneous echo pattern (BIRADS IV). A core biopsy was done and revealed invasive duct carcinoma. Automated ultrasound was superior to tomosynthesis in the characterization of this lesion by detection of the fine speculation 
Table 2 Relation between both modalities regarding the shape

\begin{tabular}{|c|c|c|c|c|c|c|c|c|c|c|}
\hline & & \multicolumn{8}{|c|}{ Digital breast tomosynthesis (DBT) shape } & \multirow{3}{*}{$p$ value } \\
\hline & & \multicolumn{2}{|l|}{ Oval } & \multicolumn{2}{|l|}{ Round } & \multicolumn{2}{|c|}{ Irregular } & \multicolumn{2}{|c|}{ No mass } & \\
\hline & & Count & $\%$ & Count & $\%$ & Count & $\%$ & Count & $\%$ & \\
\hline \multirow[t]{3}{*}{ ABUS shape } & Oval & 8 & 72.7 & 2 & 33.3 & 2 & 11.1 & 0 & .0 & \multirow[t]{3}{*}{$<0.001$} \\
\hline & Round & 1 & 9.1 & 3 & 50.0 & 0 & .0 & 0 & .0 & \\
\hline & Irregular & 2 & 18.2 & 1 & 16.7 & 16 & 88.9 & 1 & 100.0 & \\
\hline
\end{tabular}

\section{Extension of the lesion}

Extension of the lesion outside the mass margin either as speculation or satellites within more than $2 \mathrm{~cm}$.

Regarding extension of the lesion $95.2 \%$ shows no extension on both modalities and with $68.8 \%$ showing radiological extension on both studies. Five cases show extension by tomosynthesis images while non-visible by automated ultrasound.

\section{Skin thickening and retraction}

$43.5 \%$ of the malignant masses show no skin thickening on automated ultrasound while $56.5 \%$ show skin thickening.

\section{Calcification in the mass}

No calcification was visible in 21 cases with 16 cases showing calcification, 8 of them showing benign criteria, and 8 with suspicious criteria. Only two masses show calcification inside the mass. Tomosynthesis was high more sensitive in the detection of calcification than automated ultrasound.

\section{BIRADS classification}

All breast masses seen were interpreted then by the BIRADS score system.

$71.4 \%$ of benign masses were given BIRADS III by tomosynthesis, $56.2 \%$ of malignant masses were given BIRADS V while $57.1 \%$ of the benign masses were given BIRADS III by automated ultrasound, and $73.9 \%$ of malignant masses were given BIRADS V (Fig. 2).
One hundred percent agreement in BIRADS II benign lesion with 95\% agreement in malignant BIRADS V, 3 cases were given BIRADS IV by tomosynthesis images and BIRADS $\mathrm{V}$ by automated with only one appear BIRADS $\mathrm{V}$ in tomosynthesis images and IV in automated images with statistically significant results (Figs. 3 and 4).

Tomosynthesis was overestimated in $18.8 \%$ of cases and underestimated in $0 \%$ of cases while automated ultrasound was overestimated in $25 \%$ of cases and underestimated in $0 \%$ of cases as shown in Table 5 .

In detection and characterization of different breast masses sensitivity, specificity, PPV, and NPV of ABUS were $100 \%, 75 \% 84 \%, 100 \%$, respectively and in breast tomosynthesis were $100 \%, 81.25 \%, 87.5 \%$, and $100 \%$, respectively.

\section{Discussion}

Supplemental breast US or DBT is an essential additive tool for screening strategies in women at average or intermediate risk of developing breast cancer. However, early comparative studies have shown that breast US may have a benefit over DBT in women with extremely dense breasts [9].

Endo et al. [8] and Teertstra et al. [10] evaluated the diagnostic performance of digital breast tomosynthesis and full-field digital mammography and both concluded that tomosynthesis can be used as an additional technique to mammography in patients referred with an abnormal screening mammogram or with clinical symptoms.

Table 3 Relation between both modalities regarding the margins

\begin{tabular}{|c|c|c|c|c|c|c|c|c|c|c|c|c|c|c|}
\hline & & \multicolumn{12}{|c|}{ Digital breast tomosynthesis (DBT) margin } & \multirow{3}{*}{$P$ value } \\
\hline & & \multicolumn{2}{|c|}{ Circumscribed } & \multicolumn{2}{|c|}{ Obscured } & \multicolumn{2}{|c|}{ Micro lobulated } & \multicolumn{2}{|c|}{ Indistinct } & \multicolumn{2}{|c|}{ Apeculated } & \multicolumn{2}{|c|}{ No mass } & \\
\hline & & Count & $\%$ & Count & $\%$ & Count & $\%$ & Count & $\%$ & Count & $\%$ & Count & $\%$ & \\
\hline \multirow[t]{5}{*}{ ABUS margin } & Circumscribed & 11 & $84.6 \%$ & 2 & $66.7 \%$ & 0 & $.0 \%$ & 0 & $.0 \%$ & 0 & $.0 \%$ & 0 & $.0 \%$ & \multirow[t]{5}{*}{$<0.001$} \\
\hline & Angular & 0 & $.0 \%$ & 1 & $33.3 \%$ & 0 & $.0 \%$ & 1 & $33.3 \%$ & 0 & $.0 \%$ & 0 & $.0 \%$ & \\
\hline & Micro lobulated & 0 & $.0 \%$ & 0 & $.0 \%$ & 1 & $50.0 \%$ & 0 & $.0 \%$ & 1 & $6.7 \%$ & 0 & $.0 \%$ & \\
\hline & Indistinct & 1 & $7.7 \%$ & 0 & $.0 \%$ & 0 & $.0 \%$ & 2 & $66.7 \%$ & 1 & $6.7 \%$ & 1 & $100.0 \%$ & \\
\hline & Speculated & 1 & $7.7 \%$ & 0 & $.0 \%$ & 1 & $50.0 \%$ & 0 & $.0 \%$ & 13 & $86.7 \%$ & 0 & $.0 \%$ & \\
\hline
\end{tabular}


Table 4 Relation between both modalities regarding the number of masses

\begin{tabular}{|c|c|c|c|c|c|c|c|c|}
\hline & & \multicolumn{6}{|c|}{ Digital breast tomosynthesis (DBT) mass number } & \multirow{3}{*}{$p$ value } \\
\hline & & \multicolumn{2}{|l|}{ single } & \multicolumn{2}{|c|}{ multiple } & \multicolumn{2}{|l|}{ no } & \\
\hline & & Count & $\%$ & Count & $\%$ & Count & $\%$ & \\
\hline \multirow{3}{*}{$\begin{array}{c}\text { ABUS } \\
\text { mass number }\end{array}$} & Single & 16 & 72.7 & 1 & 7.1 & 1 & 100.0 & \multirow[t]{3}{*}{$<0.001$} \\
\hline & Multiple & 6 & 27.3 & 13 & 92.9 & 0 & .0 & \\
\hline & Mass & 0 & .0 & 0 & .0 & 0 & .0 & \\
\hline
\end{tabular}

A comparison of digital breast tomosynthesis and ultrasonography regarding the characterization of breast lesions was the goal of Kim et al. [11]. Results showed that digital breast tomosynthesis may provide similar reader lesion characterization performance to that of the US for breast lesions depicted on the mammogram.

On the other hand, several studies focused on automated ultrasound.
Chang et al. [12] retrospectively evaluated the detection performance of benign and malignant breast masses using 3D volume data obtained by ABUS with results showed higher performance of ABUS in the detection of malignant, large, irregular shaped masses with surrounding changes, than benign, small, or round/oval masses without surrounding changes.

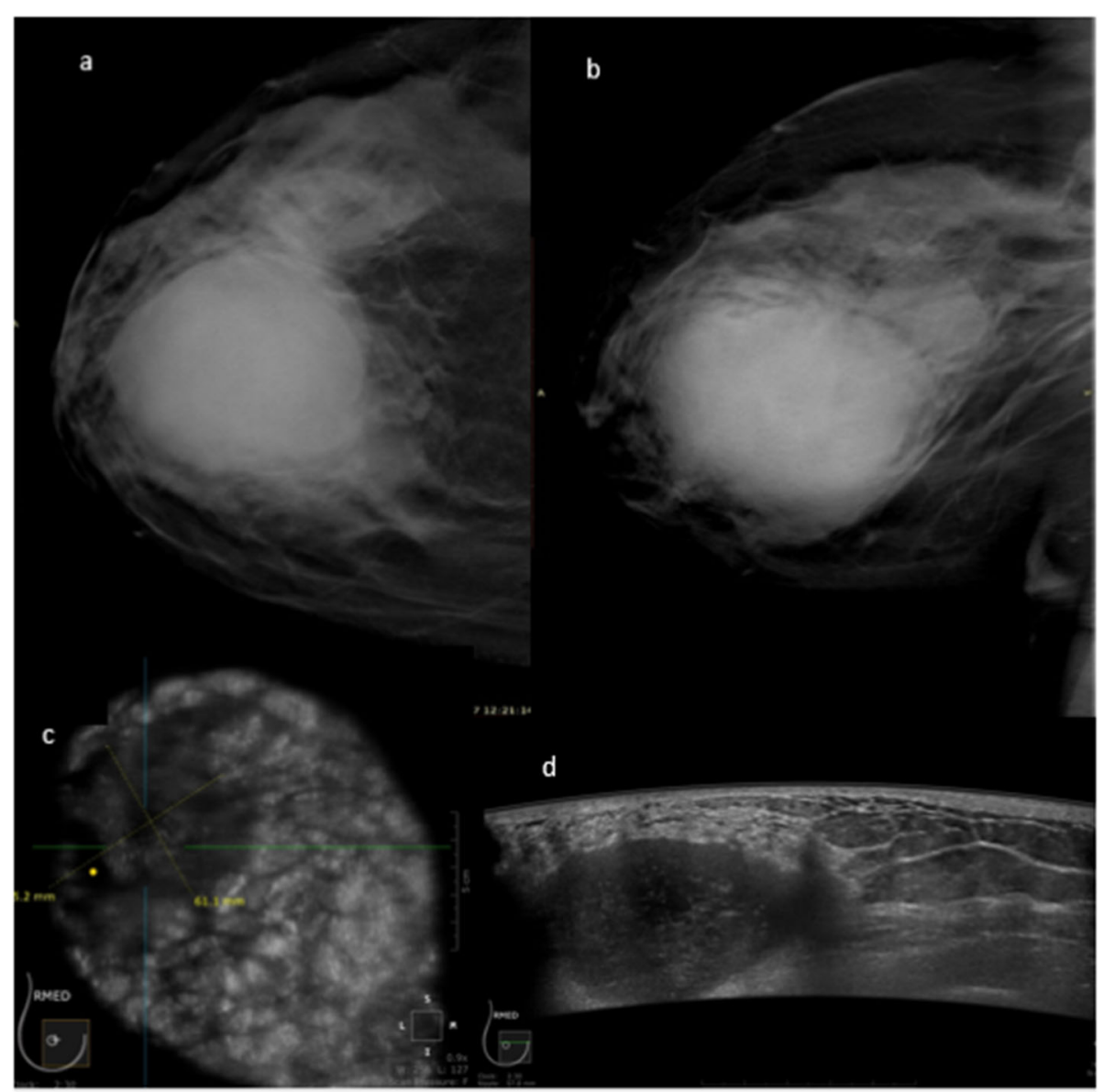

Fig. 2 A 42-year-old female patient was presented with right tender breast. Digital tomosynthesis Mx CC and MLO views of the right breast (a, b) show an oval-shaped circumscribed high-density retro areolar mass associated with skin thickening (BIRADS III). c Automated ultrasound right medial coronal image shows a large defect at 2:30 o'clock. $\mathbf{d}$ Automated ultrasound right medial axial image shows a right oval-shaped mass with indistinct margin, parallel orientation, and hypoechoic echo pattern (BIRADS III). The lesion was proved to be inflammatory mastitis with acute abscess formation by aspiration of the abscess content 


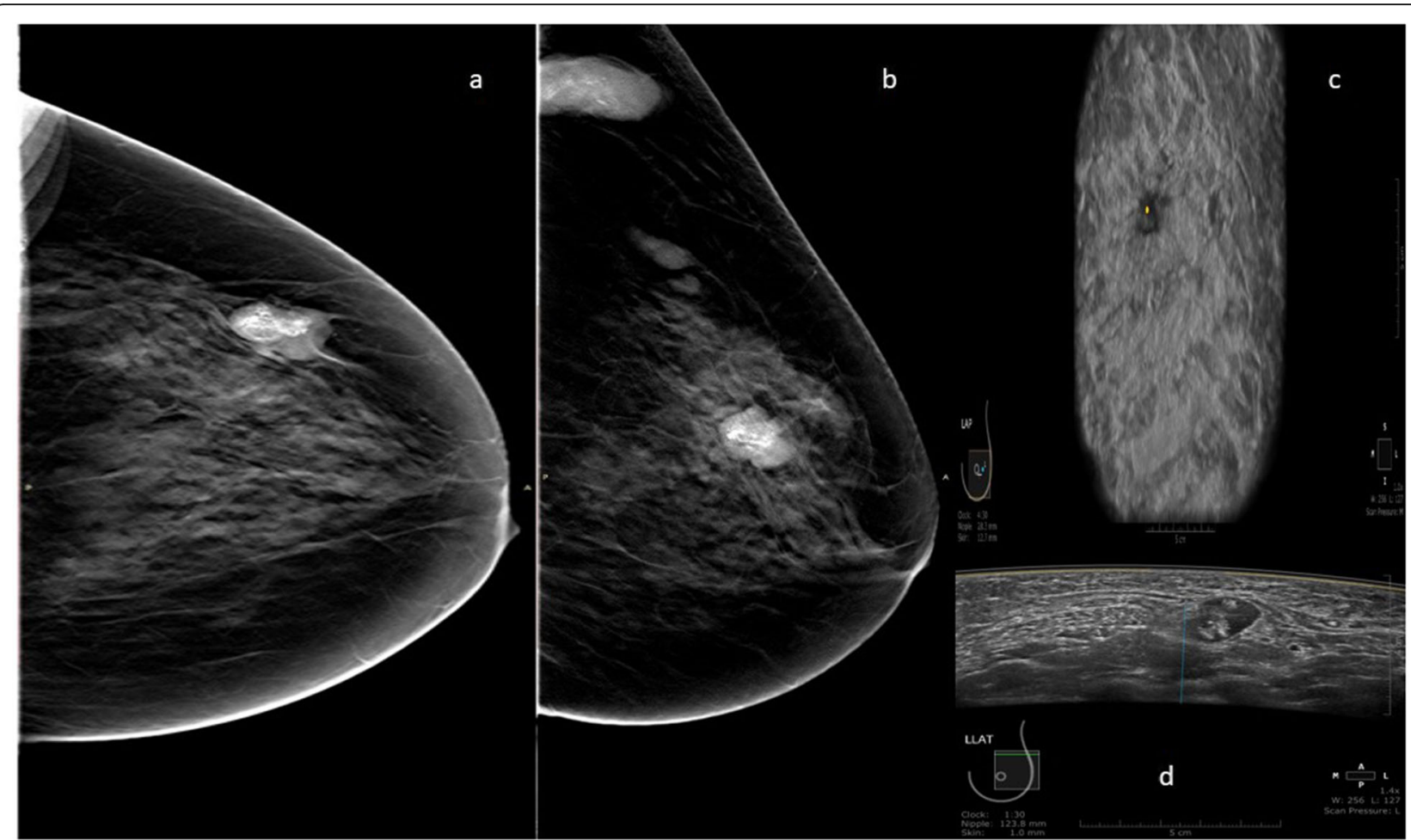

Fig. 3 A 74-year-old female patient was presented to us by a clinically palpable left breast lump. Digital mammography tomosynthesis CC and MLO views $(\mathbf{a}, \mathbf{b})$ of the left breast show an oval-shaped circumscribed high-density UOQ mass with no associated skin thickening (BIRADS IV). Automated ultrasound left AP coronal image (c) show small irregular defect at distance $28.3 \mathrm{~mm}$ from the nipple. Automated ultrasound image (d) shows a right irregular shaped mass with spiculated margins, non- parallel orientation, and heterogeneous echo pattern. (BIRADS V). A core biopsy was done and revealed adenocarcinoma. Automated ultrasound was superior to tomosynthesis in the characterization of the lesion while tomosynthesis was superior in the detection of axillary lymphadenopathy

Giuliano et al. [13] study performed in 3418 asymptomatic women with mammographically dense breasts justified the cost-benefit of implementing the use of ABUS in conjunction with mammography in the dense breast screening population study.

This study compared the diagnostic performance of automated three-dimensional (3D) breast ultrasound (US) systems and breast tomosynthesis as diagnostic tools in the work-up of mammographically detected positive findings in dense breasts.

According to the BIRADS lexicon criteria for describing malignant masses and as regards the shape, 17 masses were irregular on both modalities and were proved to be malignant, yet tomosynthesis underestimated one case since it gave the appearance of a rounded mass (normally to a benign descriptor); however, fine speculation were identified on automated ultrasound images and core biopsy proved the malignant nature of this lesion.

We found out that the sensitivity of tomosynthesis regarding the characterization of the shape of malignant masses was $69.6 \%$ while it was $87 \%$ for automated ultrasound
Regarding the margins, and as expected, $78.6 \%$ of the circumscribed masses were benign and $65 \%$ of the spiculated masses were malignant. However, two masses were circumscribed on both modalities yet pathology proved them malignant, this confirms the idea that a single criterion is not enough for radiologists to reach a correct diagnosis. Tomosynthesis was as sensitive as automated ultrasound in this study both $65.2 \%$.

Regarding the number of masses detected multiple lesions in 14 out of 19 cases with multiple masses, while automated ultrasound was able to detect the whole 19 cases.

Tomosynthesis was able to detect the extension of the mass by $43 \%$ while automated ultrasound by only $32.4 \%$.

Tomosynthesis was far better in the detection of calcification; where 16 cases were detected by tomosynthesis while automated ultrasound was only able to detect 2 cases. This is one of the situations where tomosynthesis is definitely superior over automated ultrasound. Tomosynthesis was high more sensitive in the detection of calcification than automated ultrasound matching results by Hussein et al. [14]. 


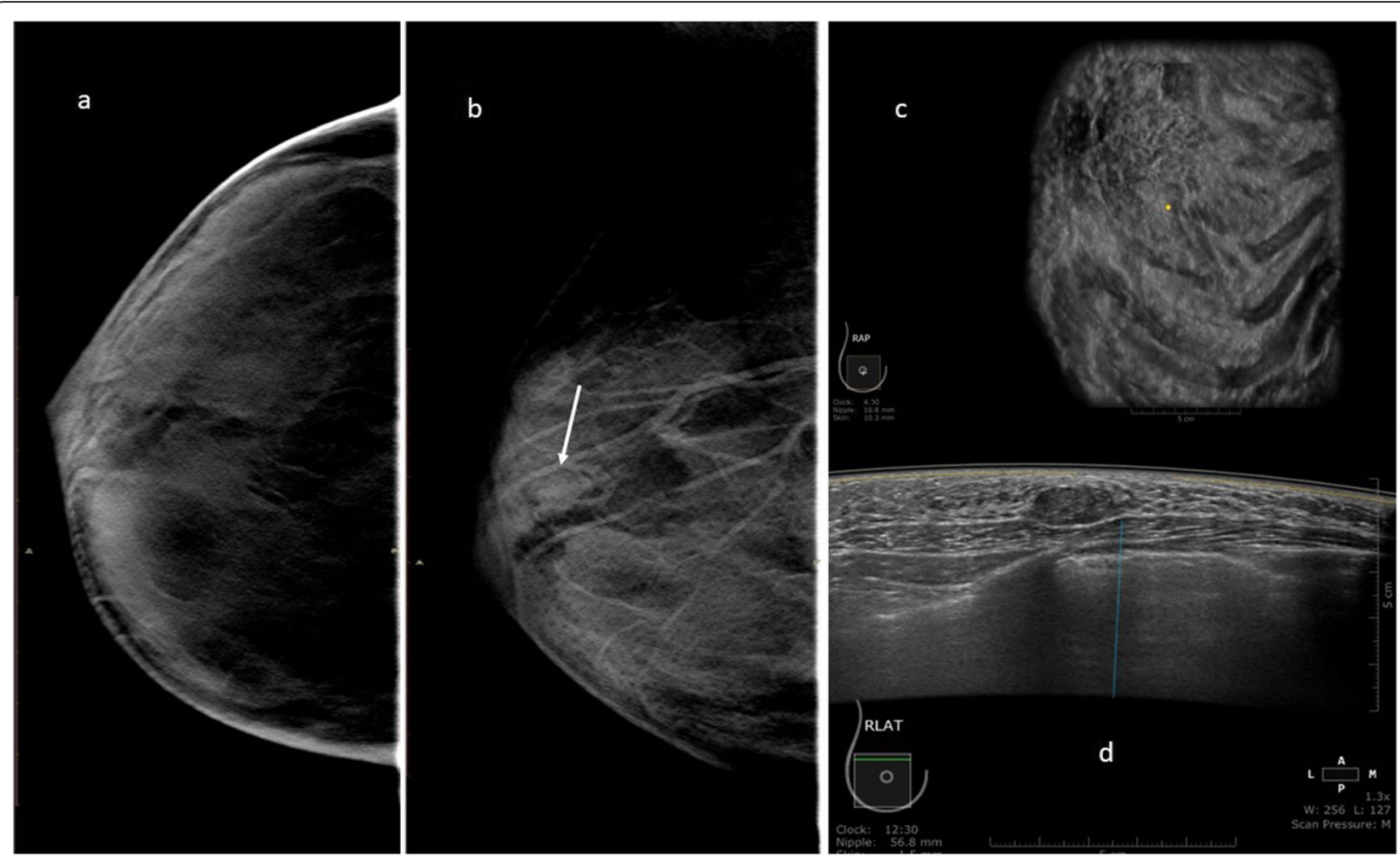

Fig. 4 A 36-year-old female patient was referred to the early detection breast clinic with a strong family history of breast cancer. Tomosynthesis CC and MLO views (a, b) of the right breast show a UOQ oval-shaped circumscribed high-density mass (BIRADS III) (arrow). c Automated ultrasound right AP coronal image showing rounded spot at distance $10.3 \mathrm{~mm}$ from the nipple. $\mathbf{d}$ Automated ultrasound right lateral image shows an oval-shaped mass with circumscribed margins, parallel orientation and hypoechoic echo pattern (BIRADS III). The lesion proved to be fibroadenoma by core biopsy. Both modalities were equal in the detection and characterization of the mass

In detection and characterization of different breast masses sensitivity, specificity, PPV, and NPV of ABUS were $100 \%, 75 \%, 84 \%$, and $100 \%$, respectively, and in breast tomosynthesis were $100 \%, 81.25 \%, 87.5 \%$, and $100 \%$, respectively.

Few studies have compared both modalities as that done by Hussein et al. [14] showing sensitivity, specificity, PPV, and NPV of ABUS was 92\%, 98\%, 92\%, and 98\%, respectively, and in BT were 92\%, 92\%, 76\%, and 98\%.

In this study, both modalities showed a higher sensitivity for the detection of breast masses both being 100\% compared to $92 \%$ in Hussein et al. study [14] but less specificity for both modalities in this study with BT showing higher specificity compared to ABUS.

Table 5 Breast masses classification according to BIRADS score system

\begin{tabular}{|c|c|c|c|c|c|c|c|c|c|c|}
\hline \multicolumn{6}{|c|}{ Digital breast tomosynthesis (DBT) } & \multicolumn{5}{|l|}{ ABUS } \\
\hline & \multicolumn{4}{|c|}{ Pathology } & \multirow{3}{*}{$p$ value } & \multicolumn{4}{|c|}{ Pathology } & \multirow{3}{*}{$p$ value } \\
\hline & \multicolumn{2}{|c|}{ Benign } & \multicolumn{2}{|c|}{ Malignant } & & \multicolumn{2}{|c|}{ Benign } & \multicolumn{2}{|c|}{ Malignant } & \\
\hline & Count & $\%$ & Count & $\%$ & & Count & $\%$ & Count & $\%$ & \\
\hline BIRADS I & 0 & 0 & 0 & 0 & $<0.001$ & 0 & 0 & 0 & 0 & $<0.001$ \\
\hline BIRADS ॥ & 3 & 21.4 & 0 & 0 & & 4 & 28.6 & 0 & 0 & \\
\hline BIRADS III & 10 & 71.4 & 0 & 0 & & 8 & 57.1 & 0 & 0 & \\
\hline BIRADS IV & 1 & 7.1 & 8 & 34.8 & & 2 & 14.3 & 6 & 26.1 & \\
\hline BIRADS V & 0 & 0 & 15 & 65.2 & & 0 & 0 & 17 & 73.9 & \\
\hline BIRADS VI & 0 & 0 & 0 & 0 & & 0 & 0 & 0 & 0 & \\
\hline Malignant & 21 & 100 & 3 & 18.8 & $<0.001$ & 21 & 100 & 4 & 25 & $<0.001$ \\
\hline Benign & 0 & 0 & 13 & 81.2 & & 0 & 0 & 12 & 75 & \\
\hline
\end{tabular}




\section{Conclusion}

The value of ABUS and DBT in the detection and characterization of breast masses were very close to one another. Since automated ultrasound provides 3D images with avoiding the radiation hazard, it can be the new era in breast cancer screening in adjacent to mammography.

\section{Abbreviations}

DBT: Digital breast tomosynthesis; ABUS: Automated breast ultrasound; 3D: Three-dimensional; US: Ultrasound; HHUS: Hand-held ultrasound; CC: Cranio-caudal; MLO: Medio-lateral oblique; PPV: Positive predictive value; NPV: Negative predictive value

\section{Acknowledgements}

Not applicable.

\section{Authors' contributions}

AM wrote the manuscript. RA collected patient data. LB image processing and collection of patient's images. MH participated in the design of the study and performed the statistical analysis. RA and LB conceived of the study, participated in its design and coordination, and helped to draft the manuscript. $\mathrm{MH}$ was responsible for revision of the draft from the clinical point of view. EG for pathology data collection and statistical analysis. All author(s) read and approved the final manuscript.

\section{Funding}

No funding sources.

\section{Availability of data and materials}

The datasets used and analyzed during the current study are available from the corresponding author on reasonable request.

\section{Ethics approval and consent to participate}

The study was approved by the ethical committee of the Faculty of Medicine, Cairo University with ethical committee approval number K-17-1122 and approval date October 2017. Informed written consent was taken from all subjects.

\section{Consent for publication}

All patients included in this research gave written informed consent to publish the data contained within this study.

\section{Competing interests}

No financial or non-financial competing interests.

\section{Author details}

'Department of Diagnostic and Interventional Radiology, Faculty of Medicine, Cairo University, Cairo, Egypt. ${ }^{2}$ Department of Diagnostic and Interventional Radiology, National Cancer Institute, Cairo University, Cairo, Egypt. ${ }^{3}$ Department of Pathology, National Cancer Institute, Cairo University, Cairo, Egypt.

Received: 16 January 2020 Accepted: 17 March 2020

Published online: 26 March 2020

\section{References}

1. Siegel RL, Miller KD, Jemal A (2019) Cancer statistics, 2019. CA Cancer J Clin 69:7-34. https://doi.org/10.3322/caac.21551

2. Etzioni R, Urban N, Ramsey S, McIntosh M, Schwartz S, Reid B, Radich J, Anderson G, Hartwell L (2003) The case for early detection. Nat Rev Cancer 3:243-252. https://doi.org/10.1038/nrc1041

3. Arleo EK, Hendrick RE, Helvie MA, Sickles EA (2017) Comparison of recommendations for screening mammography using CISNET models. Cancer 123:3673-3680. https://doi.org/10.1002/cncr.30842

4. Yaghjyan L, Colditz GA, Rosner B, Tamimi RM (2013) Mammographic breast density and subsequent risk of breast cancer in postmenopausal women according to the time since the mammogram. Cancer Epidemiol Biomarkers Prev 22:1110-1117. https://doi.org/10.1158/1055-9965.EPI-13-0169
5. Thigpen D, Kappler A, Brem R (2018) The role of ultrasound in screening dense breasts - a review of the literature and practical solutions for implementation. Diagnostics (Basel, Switzerland) 8. https://doi.org/10.3390/ diagnostics8010020

6. Chen L, Chen Y, Diao X-H, Fang L, Pang Y, Cheng A-Q, Li W-P, Wang Y (2013) Comparative study of automated breast 3-D ultrasound and handheld B-mode ultrasound for differentiation of benign and malignant breast masses. Ultrasound Med Biol 39:1735-1742. https://doi.org/10.1016/j. ultrasmedbio.2013.04.003

7. Kelly KM, Dean J, Comulada WS, Lee S-J (2010) Breast cancer detection using automated whole breast ultrasound and mammography in radiographically dense breasts. Eur Radiol 20:734-742. https://doi.org/10. 1007/s00330-009-1588-y

8. Endo T, Morita T, Oiwa M, Suda N, Sato Y, Ichihara S, Shiraiwa M, Yoshikawa K, Horiba T, Ogawa H, Hayashi Y, Sendai T, Arai T (2018) Diagnostic performance of digital breast tomosynthesis and full-field digital mammography with new reconstruction and new processing for dose reduction. Breast Cancer 25:159-166. https://doi.org/10.1007/s12282-017-0805-9

9. van Zelst JCM, Mann RM (2018) Automated three-dimensional breast US for screening: technique, artifacts, and lesion characterization. RadioGraphics 38 : 663-683. https://doi.org/10.1148/rg.2018170162

10. Teertstra HJ, Loo CE, van den Bosch MAAJ, van Tinteren H, Rutgers EJT, Muller SH, Gilhuijs KGA (2010) Breast tomosynthesis in clinical practice: initial results. Eur Radiol 20:16-24. https://doi.org/10.1007/s00330-009-1523-2

11. Kim SA, Chang JM, Cho N, Yi A, Moon WK (2015) Characterization of breast lesions: comparison of digital breast tomosynthesis and ultrasonography. Korean J Radiol 16:229-238. https://doi.org/10.3348/kjr.2015.16.2.229

12. Chang JM, Moon WK, Cho N, Park JS, Kim SJ (2011) Radiologists performance in the detection of benign and malignant masses with 3D automated breast ultrasound (ABUS). Eur J Radiol 78:99-103. https://doi.org/10.1016/j.ejrad.2011.01.074

13. Giuliano V, Giuliano C (2013) Improved breast cancer detection in asymptomatic women using 3D-automated breast ultrasound in mammographically dense breasts. Clin Imaging 37:480-486. https://doi.org/10.1016/j.clinimag.2012.09.018

14. Said NH, Adel L, Chalabi NA, ElSheikh R, Abo elmagd (2019) Automated breast ultrasound versus breast tomosynthesis in further evaluation of recalled dense breasts after screening mammograms ECR 2019: Book of Abstracts. Insights Imaging 10:22 . doi: https://doi.org/10.1186/s13244-019-0713-y

\section{Publisher's Note}

Springer Nature remains neutral with regard to jurisdictional claims in published maps and institutional affiliations.

\section{Submit your manuscript to a SpringerOpen ${ }^{\circ}$ journal and benefit from:}

- Convenient online submission

- Rigorous peer review

- Open access: articles freely available online

High visibility within the field

- Retaining the copyright to your article

Submit your next manuscript at $>$ springeropen.com 